\title{
Calculating exclusion limits for Weakly Interacting Massive Particle direct detection experiments without background subtraction
}

\author{
Anne M. Green \\ Astronomy Unit, School of Mathematical Sciences, Queen Mary University of London, \\ Mile End Road, London, E1 4NS, U. K.
}

(October 28, 2018)

\begin{abstract}
Competitive limits on the weakly interacting massive particle (WIMP) spin-independent scattering cross section are currently being produced by ${ }^{76} \mathrm{Ge}$ detectors originally designed to search for neutrinoless double beta decay, such as the Heidelberg-Moscow and IGEX experiments. In the absence of background subtraction, limits on the WIMP interaction cross section are set by calculating the upper confidence limit on the theoretical event rate, given the observed event rate. The standard analysis technique involves calculating the $90 \%$ upper confidence limit on the number of events in each bin, and excluding any set of parameters (WIMP mass and cross-section) which produces a theoretical event rate for any bin which exceeds the $90 \%$ upper confidence limit on the event rate for that bin. We show that, if there is more than one energy bin, this produces exclusion limits that are actually at a lower degree of confidence than $90 \%$, and are hence erroneously tight. We formulate criteria which produce true $90 \%$ confidence exclusion limits in these circumstances, including calculating the individual bin confidence limit for which the overall probability that no bins exceeds this confidence limit is $90 \%$ and calculating the $90 \%$ minimum confidence limit on the number of bins which exceed their individual bin $90 \%$ confidence limits. We then compare the limits on the WIMP cross-section produced by these criteria with those found using the standard technique, using data from the Heidelberg-Moscow and IGEX experiments.
\end{abstract}

PACS numbers: 98.80Cq, 14.80Ly

astro-ph/yymmddd

\section{INTRODUCTION}

The non-Keplerian rotation curves of spiral galaxies imply that, provided that Newtonian gravity is correct, they are surrounded by halos of dark matter [1]. The nature of the dark matter is unknown [2], with possible candidates including MAssive Compact Halo Objects (MACHOs), such as brown dwarves, Jupiters or black holes, and elementary particles, known as Weakly Interacting Massive Particles (WIMPs). Currently the best motivated WIMP candidate is the neutralino, the lightest supersymmetric particle 3 .

Neutralinos, and WIMPs generically, can potentially be directly detected via their elastic scattering off target nuclei. Over the past five years the DAMA collaboration has found an annual modulation in the signal in their $\mathrm{NaI}$ detector, consistent with nuclear recoils caused by WIMPs with mass $m_{\chi} \sim 54 \mathrm{GeV}$. Their claim has been met with some scepticism [5], due to the difficulties involved in extracting the small ( $\sim$ a few per-cent) annual modulation signal from the backgrounds present, along with various technical concerns. It is therefore crucial to verify this result using other detectors and techniques.

Currently the most stringent spin-independent WIMP exclusion limits come from the CDMS experiment's 10.6 $\mathrm{kgd}$ exposure at Stanford [6]. They use the measured nuclear recoils in Si detectors (which are more sensitive to neutrons than to WIMPs), along with the multiple scattering events in their Ge detectors, to subtract the neutron background from their Ge single scatter events.
They find, using a sophisticated likelihood analysis along with Monte-Carlo simulations (see Ref. [7] for details), that the Ge nuclear recoil events observed are most likely due to neutrons rather than WIMPs. The CDMS experiment will be moving to the Soudan mine, where the lower backgrounds should, in the long term, allow the improvement of the constraints on the WIMP cross-section by several orders of magnitude [8].

Competitive constraints are also coming from ${ }^{76} \mathrm{Ge}$ detectors originally designed to search for neutrinoless double beta decay, such as the Heidelberg-Moscow [9] and IGEX [10] experiments. The Heidelberg-Moscow experiment operates five ${ }^{76} \mathrm{Ge}$ detectors with total active mass of $10.96 \mathrm{~kg}$, and to date have analysed data taken over 0.249 years, from one detector with an active mass of $2.758 \mathrm{~kg}$ and $86 \%$ enrichment in ${ }^{76} \mathrm{Ge}$, giving a total exposure of $250.836 \mathrm{kgd}$. The IGEX collaboration have to date analysed data taken over 30 days by one of their enriched detectors with an active mass of $2.0 \mathrm{~kg}$, giving a total exposure of $60.0 \mathrm{kgd}$. In this paper we examine the method used to calculate exclusion limits from these experiments. We outline the calculation of upper confidence limits on the number of events per bin, and formulate criteria which produce true overall $90 \%$ confidence exclusion limits on the WIMP scattering crosssection. We then compare the exclusion limits on the cross-section calculated using these criteria with those calculated using the standard analysis employed by the Heidelberg-Moscow and IGEX collaborations. 


\section{CONFIDENCE LIMIT CALCULATION}

In experiments without background subtraction, such as Heidelberg-Moscow (HM) [9] and IGEX [10], no constraints on the WIMP parameters can be derived from the shape of the observed energy spectrum. However any set of WIMP parameters (mass and cross-section) which would produce more events than are observed, at some confidence level, can be excluded at that confidence level. The probability of finding an observed number of events $N_{\text {obs }}$ given an expected number of events $\lambda$ is given by the Poisson distribution:

$$
P\left(N_{\mathrm{obs}} \mid \lambda\right)=\frac{e^{-\lambda} \lambda^{N_{\mathrm{obs}}}}{N_{\mathrm{obs}} !},
$$

where the 'l' denotes 'given'. Note that whilst $N_{\text {obs }}$ is, by definition, an integer, $\lambda$ can take any positive value. Using Bayes theorem

$$
\begin{aligned}
P\left(\lambda \mid N_{\text {obs }}\right) & =\frac{P\left(N_{\text {obs }} \mid \lambda\right) P(\lambda)}{P\left(N_{\text {obs }}\right)} \\
& =\frac{P\left(N_{\text {obs }} \mid \lambda\right) P(\lambda)}{\int_{0}^{\infty} P\left(N_{\text {obs }} \mid \lambda\right) P(\lambda) \mathrm{d} \lambda},
\end{aligned}
$$

where $P(\lambda)$ is the prior probability distribution for $\lambda$. Following the Particle Data Group we take $P(\lambda)$ to be uniform in $\lambda$ 用 and the above expression then simplifies to

$$
P\left(\lambda \mid N_{\mathrm{obs}}\right)=P\left(N_{\mathrm{obs}} \mid \lambda\right) \text {. }
$$

It then follows that the probability that $\lambda$ is greater than some value $\lambda_{\mathrm{p}}$ is

$$
\begin{aligned}
P\left(\lambda>\lambda_{\mathrm{p}} \mid N_{\text {obs }}\right) & =\int_{\lambda_{p}}^{\infty} P\left(\lambda \mid N_{\text {obs }}\right) \mathrm{d} \lambda \\
& =\int_{\lambda_{\mathrm{p}}}^{\infty} \frac{e^{-\lambda} \lambda^{N_{\text {obs }}}}{N_{\text {obs }} !} \mathrm{d} \lambda .
\end{aligned}
$$

Using the relation 12

$$
\int_{u}^{\infty} x^{n} e^{-\mu x}=e^{-u \mu} \sum_{k=0}^{n} \frac{n !}{k !} \frac{u^{k}}{\mu^{n-k+1}},
$$

we find that

$$
\begin{aligned}
P\left(\lambda>\lambda_{\mathrm{p}} \mid N_{\text {obs }}\right) & =e^{-\lambda_{\mathrm{p}}} \sum_{n=0}^{N_{\text {obs }}} \frac{\left(\lambda_{\mathrm{p}}\right)^{n}}{n !} \\
& =P\left(N \leq N_{\text {obs }} \mid \lambda_{\mathrm{p}}\right) .
\end{aligned}
$$

The HM and IGEX collaboration bin their data in bins of width $1 \mathrm{keV}$ (42 bins from $9 \mathrm{keV}$ to $51 \mathrm{keV}$ for HM

\footnotetext{
*See Ref. 11] and references therein for a contrary view however.
}

and 45 bins from $4 \mathrm{keV}$ to $49 \mathrm{keV}$ for IGEX). In their analysis [9, 10], the $90 \%$ confidence exclusion limit on the cross-section, $\sigma_{\mathrm{p}}$, is calculated by finding for each $m_{\chi}$ the value of $\sigma_{\mathrm{p}}$ for which the theoretical event rate in one of the bins just exceeds the $90 \%$ bin confidence limit on the event rate in that bin. The Heidelberg-Moscow collaboration employ a slight variation of this technique, re-binning the data in overlapping $5 \mathrm{keV}$ bins (i.e. 9-14 $\mathrm{keV}, 10-15 \mathrm{keV}$ etc.), claiming that otherwise the limits obtained are too conservative [9].

By definition a $90 \%$ upper confidence limit means that there is a $10 \%$ probability that a theoretical number of events greater than the $90 \%$ confidence limit produced the observed number of events, for any given bin. We will now consider an ensemble of $N_{\mathrm{t}}$ bins. The probability distibution of the number of bins for which the theoretical number of events exceeds the $90 \%$ bin confidence limit, $N_{\mathrm{e}}$, is given by

$$
\begin{aligned}
P\left(N_{\mathrm{e}}>0\right) & =1-P\left(N_{\mathrm{e}}=0\right)=1-(0.9)^{N_{\mathrm{t}}} \\
& >0.1 \quad \text { if } N_{\mathrm{t}}>1 .
\end{aligned}
$$

Therefore the exclusion limits found using the standard analysis actually correspond to a lower degree of confidence than $90 \%$ and are hence erroneously tight. The larger the total number of bins the larger the discrepancy will be. For an ensemble of bins the probability that the observed number of events in $N_{\mathrm{e}}$ of the bins was produced by a theoretical number of events greater than the $100 \mathrm{p} \%$ bin upper confidence limit is given by the binomial distribution:

$$
P\left(N_{\mathrm{e}}\right)=C_{N_{\mathrm{e}}}^{N_{\mathrm{t}}} \mathrm{p}^{N_{\mathrm{e}}}(1-\mathrm{p})^{N_{\mathrm{t}}-N_{\mathrm{e}}},
$$

where

$$
C_{N_{\mathrm{e}}}^{N_{\mathrm{t}}}=\frac{N_{\mathrm{t}} !}{N_{\mathrm{e}} !\left(N_{\mathrm{t}}-N_{\mathrm{e}}\right) !},
$$

provided that each bin is independent (this is not the case for the HM experiment's overlapping $5 \mathrm{keV}$ bins).

We will now use the probability distribution of the number of bins $N_{\mathrm{e}}$ exceeding their $100 \mathrm{p} \%$ bin confidence limit (eq. (8) to formulate criteria which produce true 90\% minimum confidence exclusion limits. Firstly, for any given total number of bins, we can find the confidence level $\mathrm{cl}$ for which the probability that none of the bins exceed their $100 \mathrm{cl} \%$ bin confidence is $90 \%$ :

$$
C_{0}^{N_{\mathrm{t}}}(1-\mathrm{cl})^{N_{\mathrm{t}}}=0.1
$$

\footnotetext{
${ }^{\dagger}$ Whilst the values of $\lambda$ predicted for each bin by an underlying theoretical model are not independent of each other, the number of events observed in each bin is an independent Poisson process, governed by the underlying value of $\lambda$ for that bin.
} 
We can also calculate the $90 \%$ minimum upper confidence limit on the number of bins which exceed their $90 \%$ bin confidence limit. We define $N_{90}$ as the smallest integer which satisfies:

$$
\sum_{N_{\mathrm{e}}=N_{90}}^{N_{\text {tot }}}\left[C_{N_{\mathrm{e}}}^{N_{\mathrm{t}}} 0.1^{N_{\mathrm{e}}}(0.9)^{\left(N_{\mathrm{t}}-N_{\mathrm{e}}\right)}\right]<0.1 .
$$

Any set of WIMP parameters which produces $N_{\mathrm{e}} \geq N_{90}$ is excluded at at least $90 \%$ confidence. For $N_{\mathrm{t}}=42$ or $45, N_{90}=8$ and $P\left(N_{\mathrm{e}}<N_{90}\right)=0.946(0.924)$ for $N_{\mathrm{t}}=$ 42 (45). Since $N_{\mathrm{e}}$ can only take on integer values this criteria does not produce exact $90 \%$ exclusion limits, but the amount by which the confidence limits are stronger than $90 \%$ is known, and fixed for fixed $N_{\mathrm{t}}$.

A simple minded way to avoid the problem of calculating $90 \%$ overall confidence limits would be to discard all but one of the energy bins; $N_{\mathrm{t}}=1$ then and the $90 \%$ bin confidence limit gives an overall $90 \%$ confidence limit. The obvious choice for which energy bin to use is the lowest, threshold, energy bin, since $\mathrm{d} R / \mathrm{d} E_{\mathrm{R}}$ decreases exponentially with increasing $E_{\mathrm{R}}$ for the standard Maxwellian halo model. It is likely that this wasteful method will produce weaker exclusion limits than can be found using the entire data set though.

\section{EXCLUSION LIMITS}

We will now calculate the exclusion limits resulting from the HM and IGEX data, assuming a standard Maxwellian halo, using a variety of criteria: the HM collaboration's overlapping $5 \mathrm{keV}$ bins technique (criteria $\mathrm{A}-\mathrm{HM}$ only), no bins exceeding their $90 \%$ bin confidence limit (criteria B), no bins exceeding their 100cl\% bin confidence limit (criteria C), less than $N_{90}=8$ bins exceeding their $90 \%$ bin confidence limit (criteria D) and from the lowest energy bin not exceeding its $90 \%$ bin confidence limit (criteria E). The bin confidence limit cl for criteria $\mathrm{C}$ has been calculated from the binomial distribution to give $90 \%$ overall (as opposed to individual bin) exclusion limits; for the $N_{\mathrm{t}}=42$ bins of the HM experiment $\mathrm{cl}=0.9975$ whilst for the $N_{\mathrm{t}}=45$ bins of the IGEX experiment $\mathrm{cl}=0.9977$. In Section II we found that Criteria D produces $94.6 \%$ confidence exclusion limits for the HM experiment and $92.4 \%$ confidence exclusion limits for the IGEX experiment. Note that $\lambda_{\mathrm{p}} / N_{\mathrm{obs}}$, for any fixed p, decreases with increasing $N_{\text {obs }}$; the smaller the number of counts observed the greater the uncertainty in the underlying see e.g. Refs. [3, 13, 9, 10]. theoretical number of counts. For details of the calculation of the theoretical event rate The resulting exclusion limits are plotted in Fig. 1, and in Tables I and II we list the energy bins from which the exclusion limits on $\sigma_{\mathrm{p}}$ arise, as a function of $m_{\chi}$, for each criteria.

The constraints from criteria A and B are similar and are tighter than those found using either of the crite-
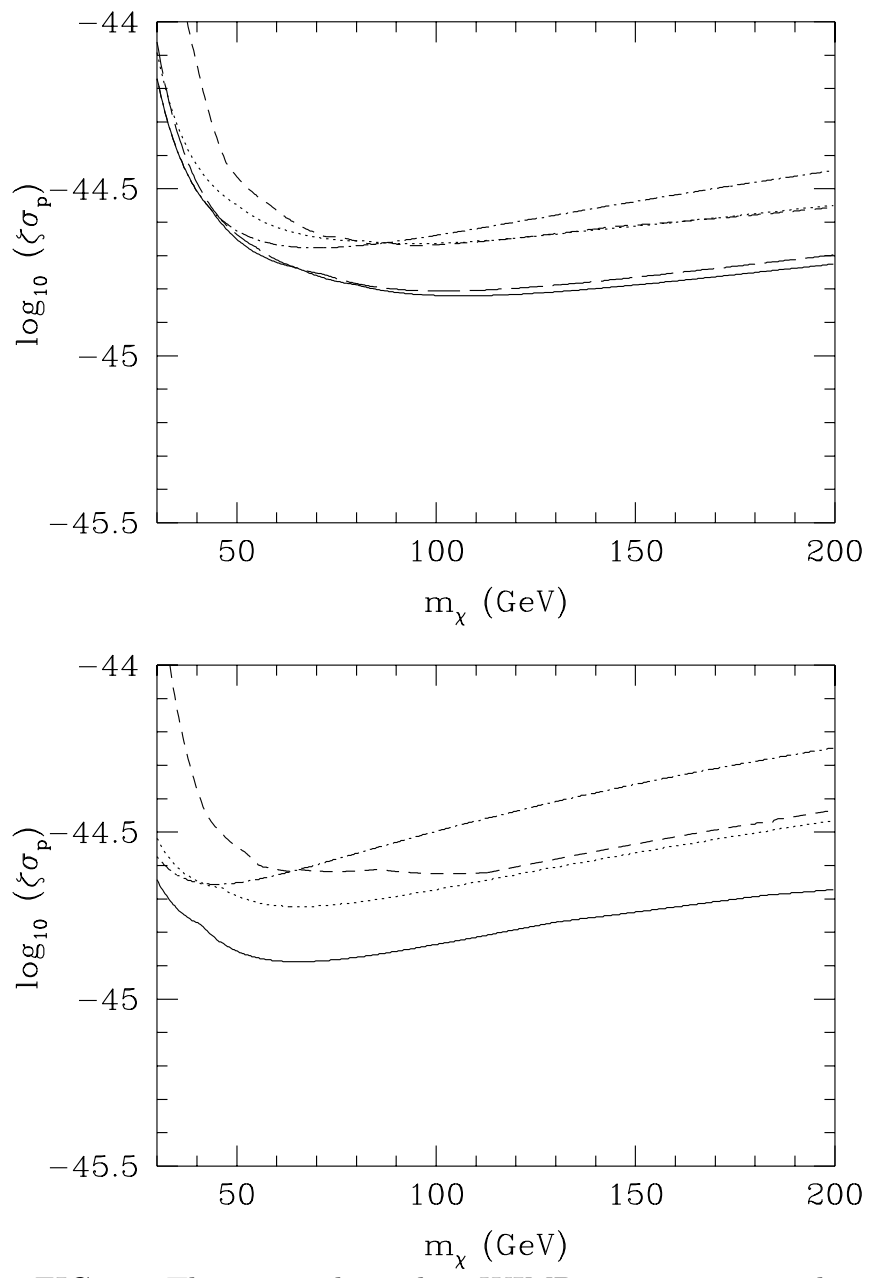

FIG. 1. The spin-independent WIMP cross-section exclusion limits found from the HM (top panel) and IGEX (lower panel) data, assuming the standard Maxwellian halo model, from: using the sliding $5 \mathrm{keV}$ bins method (long dashed line), no bin exceeding its $90 \%$ bin confidence limit (solid), no bin exceeding its $99.75 \%$ bin confidence limit (dotted), no more than 7 bins exceeding their $90 \%$ bin confidence limit (short dashed) and the lowest energy bin not exceeding its $90 \%$ bin confidence limit (dot-dashed)

ria (C and $\mathrm{D})$ calculated from the binomial distribution to give $90 \%$ overall confidence limits, by roughly $\Delta\left[\log _{10}(\zeta \sigma)\right] \sim 0.2$. As shown above $($ Eq. (7) $)$ the requirement that no bin exceeds its $90 \%$ bin confidence limit (i.e. criteria B) actually produces exclusion limits at $1.2 \%(0.9 \%)$ confidence for $N_{\mathrm{t}}=42(45)$ bins. Criteria $\mathrm{D}$ produces significantly weaker constraints than $\mathrm{C}$ for $m_{\chi} \lesssim 80 \mathrm{GeV}$, and we will discuss the reason for this below. For $m_{\chi} \gtrsim 80 \mathrm{GeV}$ the exclusion limit from criteria $\mathrm{D}$ is slightly weaker than that from criteria $\mathrm{C}$ since criteria D produces stronger than $90 \%$ confidence exclusion limits (94.6\% for HM and $92.4 \%$ for IGEX). For the HM data criteria $\mathrm{E}$ produces exclusion limits which are identical to those from Criteria B for small $m_{\chi}$, where the lowest energy bin is the most constraining, for large 


\begin{tabular}{|c|c|}
\hline \hline$m_{\chi}(\mathrm{GeV})$ & Energy bin(s) \\
\hline \multicolumn{2}{|c|}{ Criteria A } \\
\hline$<54$ & $9-14 \mathrm{keV}$ \\
$54-72$ & $11-16 \mathrm{keV}$ \\
$>72$ & $15-20 \mathrm{keV}$ \\
\hline \multicolumn{2}{|c|}{ Criteria B } \\
\hline$<44$ & $9-10 \mathrm{keV}$ \\
$44-65$ & $11-12 \mathrm{keV}$ \\
$65-81$ & $15-16 \mathrm{keV}$ \\
$>81$ & $18-19 \mathrm{keV}$ \\
\hline \multicolumn{2}{|c|}{ Criteria C } \\
\hline $51-65$ & $9-10 \mathrm{keV}$ \\
$65-83$ & $11-12 \mathrm{keV}$ \\
$83-142$ & $12-13 \mathrm{keV}$ \\
$>142$ & $15-16 \mathrm{keV}$ \\
\hline \multicolumn{2}{|c|}{$18-19 \mathrm{keV}$} \\
\hline$<47$ & Criteria D \\
$47-72$ & 1234567 \\
$72-83$ & 1234610 \\
$83-93$ & 13467810 \\
$>93$ & 34678910 \\
\hline \hline
\end{tabular}

TABLE I. The most constraining energy bins for the Heidelberg-Moscow data, for the criteria described in the text, for a standard Maxwellian halo.

$m_{\chi}$ it produces the weakest exclusion limit since the more constraining higher energy bins have been discarded. For the IGEX data it turns out that the lowest energy bin is never the most constraining, and even for small $m_{\chi}$ criteria E produces weaker limits than Criteria B.

For both data sets the number of events per bin initially decreases fairly sharply with increasing energy $\left(E_{\mathrm{V}} \lesssim 18(11) \mathrm{keV}\right.$ for HM (IGEX)). For larger energies the number of events per bin varies between 2-17 (0-10) for HM (IGEX). For the standard Maxwellian halo $\mathrm{d} R / \mathrm{d} E_{\mathrm{R}}$ decreases exponentially with increasing $E_{\mathrm{R}}$. The decrease is steepest for small $m_{\chi}$, with $\mathrm{d} R / \mathrm{d} E_{\mathrm{R}}\left(E_{\mathrm{R}} \rightarrow 0\right)$ being largest for small $m_{\chi}$. For small $m_{\chi}$ the theoretical energy spectrum falls off more rapidly than the observed energy spectrum and the exclusion limit comes from the lowest energy bins, where the observed number of events is largest. The larger the observed number of events the smaller the difference between $\lambda_{90}$ and $\lambda_{\mathrm{cl}}$, and hence the difference in the exclusion limits produced by criteria $\mathrm{B}$ and $\mathrm{C}$ is smallest for small $m_{\chi}$. For a rapidly decreasing theoretical energy spectrum $\sigma_{\mathrm{p}}$ has to be so large for eight bins to exceed their bin confidence limit that the theoretical event rate in the lowest bin is far greater than $\lambda_{\mathrm{cl}}$, and criteria D produces misleadingly weak exclusion limits.

If the WIMP velocity distribution is not close to Maxwellian, then the form of $\mathrm{d} R / \mathrm{d} E_{\mathrm{R}}$ will deviate from exponential and the error in the exclusion limits produced by the standard analysis technique could be more

\begin{tabular}{|c|c|}
\hline$m_{\chi}(\mathrm{GeV})$ & Energy bin(s) \\
\hline \multicolumn{2}{|c|}{ Criteria B } \\
\hline$<41$ & $5-6 \mathrm{keV}$ \\
\hline $41-131$ & $8-9 \mathrm{keV}$ \\
\hline $131-186$ & $14-15 \mathrm{keV}$ \\
\hline$>186$ & $24-25 \mathrm{keV}$ \\
\hline \multicolumn{2}{|c|}{ Criteria C } \\
\hline$<47$ & $5-6 \mathrm{keV}$ \\
\hline$>47$ & $8-9 \mathrm{keV}$ \\
\hline \multicolumn{2}{|c|}{ Criteria D } \\
\hline$<42$ & 1234567 \\
\hline $42-56$ & 12345611 \\
\hline $56-57$ & 1235101112 \\
\hline $57-63$ & 1245101112 \\
\hline $63-79$ & 2456101112 \\
\hline $79-114$ & 24510111221 \\
\hline $114-187$ & $\begin{array}{lllllll}2 & 5 & 10 & 11 & 12 & 14 & 21\end{array}$ \\
\hline$>187$ & $\begin{array}{lllllll}4 & 5 & 10 & 11 & 12 & 14 & 21\end{array}$ \\
\hline
\end{tabular}

TABLE II. The most constraining energy bins for the IGEX data, for the criteria described in the text, for a standard Maxwellian halo.

significant. As an example we will study the exclusion limits for Sikivie's late infall model [14], where the CDM distribution at the Earth's location consists of a number of velocity flows plus a smooth isothermal background distribution. The resulting WIMP differential event rate $\mathrm{d} R / \mathrm{d} E_{\mathrm{R}}$ has a series of steps and falls off more rapidly than that produced by a pure Maxwellian halo [15]. Whilst it is unlikely that such velocity flows are present in the Milky Way [16], the real velocity distribution produced by the hierarchical formation of the galactic halo is likely to deviate significantly from a smooth Maxwellian distribution, resulting in a $\mathrm{d} R / \mathrm{d} E_{\mathrm{R}}$ which varies significantly from the exponential decline produced by the standard Maxwellian halo. The parameters of the late infall model can be found in Ref. [15], the total local halo density is $\rho_{\chi}=0.57 \mathrm{GeV} \mathrm{cm}^{-3}$ which corresponds to $\zeta=1.9$. In Fig. 2 we plot the exclusion limits found from the HM data for this model, using the criteria defined above. In table III we list the bins from which the limit on $\sigma_{\mathrm{p}}$ arises for each criteria, as a function of $m_{\chi}$.

For $m_{\chi} \lesssim 50 \mathrm{GeV}$ the difference in the exclusion limits produced by the different criteria is relatively small. As $m_{\chi}$ is increased the step-like drops in $\mathrm{d} R / \mathrm{d} E_{\mathrm{R}}$ caused by the velocity flows move to higher $E_{\mathrm{R}}[15]$, producing a sudden change in the predicted number of events for some of the bins, changing the bin from which the constraint on $\sigma_{\mathrm{p}}$ originates and, for criteria $\mathrm{B}$ and $\mathrm{C}$, producing a kink in the exclusion limit curve. Criteria B produces limits which are tighter than those from Criteria $\mathrm{C}$ by roughly $\Delta\left[\log _{10}(\zeta \sigma)\right] \sim 0.2$, with the difference decreasing with decreasing $m_{\chi}$, once more. For criteria $\mathrm{C}$ the cusps in the exclusion limit are shifted to slightly higher $m_{\chi}, \Delta m_{\chi} \sim 5 \mathrm{GeV}$ for $m_{\chi} \gtrsim 80 \mathrm{GeV}$, since $\lambda_{\mathrm{cl}} / \lambda_{90}$ in- 


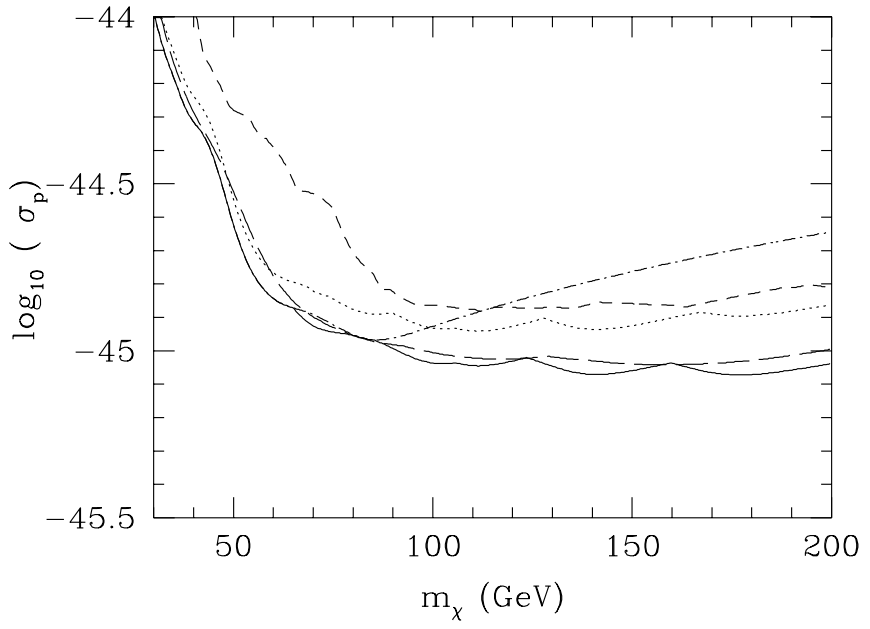

FIG. 2. The spin-independent WIMP cross-section exclusion limits found for Sikivie's late infall model from the HM data found from: the sliding $5 \mathrm{keV}$ bins method (long dashed line), no bin exceeding its $90 \%$ bin confidence limit (solid), no bin exceeding its $99.75 \%$ bin confidence limit (dotted), no more than 7 bins exceeding the $90 \%$ bin confidence limit (short dashed) and the lowest energy bin not exceeding its $90 \%$ bin confidence limit (dot-dashed).

creases with decreasing $N_{\text {obs }}$. Once more criteria D produces weaker exclusion limits then criteria $\mathrm{C}$ for $m_{\chi} \lesssim 80$ $\mathrm{GeV}$, although for this halo model the difference between criteria $\mathrm{C}$ and $\mathrm{D}$ at large $m_{\chi}$, is larger than for the standard halo model. The cusps in the exclusion limits are smoothed out to some extent by criteria A and D since for these criteria the limits arise from a range of energies (a $5 \mathrm{keV}$ bins for $\mathrm{A}$ and $71 \mathrm{keV}$ bins for $\mathrm{D}$ ), rather than a single $1 \mathrm{keV}$ bin.

\section{CONCLUSIONS}

We have shown that the standard analysis technique for calculating exclusion limits from WIMP direct detection experiments without background subtraction produces exclusion limits that are actually at a lower degree of confidence than the stated $90 \%$, and are hence erroneously tight, if there is more than one energy bin.

We have examined criteria which produce true $90 \%$ minimum confidence exclusion limits for an ensemble of bins. The first (criteria $\mathrm{C}$ above) involves calculating the individual bin confidence limit for which the overall probability that no bin exceeds this confidence limit is $90 \%$. For the 42 bins of the HM experiment this individual bin limit is $99.75 \%$ whilst for the 45 bins of the IGEX experiment it is $99.77 \%$. The second (D above) involves calculating the minimum $90 \%$ upper confidence limit on the number of bins which exceed their individual bin $90 \%$ confidence limit, for 42 or 45 bins this limit is 8. Finally the third (E above) involves using only the lowest, threshold, energy bin.

\begin{tabular}{|c|c|}
\hline$m_{\chi}(\mathrm{GeV})$ & Energy bins \\
\hline \multicolumn{2}{|c|}{ Criteria A } \\
\hline$<93$ & 9-14 keV \\
\hline $93-130$ & $11-16 \mathrm{keV}$ \\
\hline $130-141$ & $14-19 \mathrm{keV}$ \\
\hline$>141$ & $15-20 \mathrm{keV}$ \\
\hline \multicolumn{2}{|c|}{ Criteria B } \\
\hline$<65,80-85$ & $9-10 \mathrm{keV}$ \\
\hline $65-7885-106$ & $11-12 \mathrm{keV}$ \\
\hline $78-80$ 106-124 & $12-13 \mathrm{keV}$ \\
\hline $124-160$ & $15-16 \mathrm{keV}$ \\
\hline$>160$ & $18-19 \mathrm{keV}$ \\
\hline \multicolumn{2}{|c|}{ Criteria $\mathrm{C}$} \\
\hline$<68,73-89$ & $9-10 \mathrm{keV}$ \\
\hline $68-73,89-106$ & $11-12 \mathrm{keV}$ \\
\hline $106-128$ & $12-13 \mathrm{keV}$ \\
\hline $128-167$ & $15-16 \mathrm{keV}$ \\
\hline$>167$ & $18-19 \mathrm{keV}$ \\
\hline \multicolumn{2}{|c|}{ Criteria D } \\
\hline$<43,46-47,66-87$ & 1234567 \\
\hline $43-46,48-59,87-96,108-113$ & 12346710 \\
\hline $47-48,59-66$ & 12345710 \\
\hline $96-108$ & 12347810 \\
\hline $113-116$ & 23456710 \\
\hline $116-135$ & 34567810 \\
\hline $135-163$ & 34678910 \\
\hline $163-190$ & 467891011 \\
\hline$>190$ & 4789101112 \\
\hline
\end{tabular}

TABLE III. The most constraining energy bins for the Heidelberg-Moscow data, for the criteria described in the text, for Sikivie's late infall halo model.

We then compared the exclusion limits produced by these criteria with those produce by the standard analysis technique, for both the HM and IGEX experiments for a standard Maxwellian halo, and for the HM experiment for Sikivie's late infall model. We found that the standard technique produces limits which are erroneously tighter by roughly $\Delta\left[\log _{10}(\zeta \sigma)\right] \sim 0.2$. for $m_{\chi} \gtrsim 80 \mathrm{GeV}$. The difference would be larger for experiments with larger numbers of bins and/or less events in the most constraining bins. Criteria D produces exclusion limits which are misleadingly weak for small $m_{\chi}$ and does not produce exactly $90 \%$ exclusion limits, since the number of bins is an integer. Criteria $\mathrm{E}$ is wasteful and produces substantially weaker exclusion limits than could be found using the entire data set. We therefore recommend that criteria $\mathrm{C}$ should be used to produce overall $90 \%$ confidence limits.

For halo models with a significant Maxwellian component $\mathrm{d} R / \mathrm{d} E_{\mathrm{R}}$ decreases roughly exponentially with increasing $E_{\mathrm{R}}$, with the decrease being steepest for small $m_{\chi}$. In this case, for both the HM and IGEX data, for small $m_{\chi}$ the lowest energy bins, which have the highest numbers of observed events, are most constraining, 
whilst for larger $m_{\chi}$ the higher energy bins are the most constraining. Care therefore needs to be taken to produce overall 90\% confidence limits. Using upper confidence limits on the number of events per bin to produce exclusion limits for experiments without background subtraction appears to be more involved than previously realised, and more sophisticated analysis techniques need to be developed. Since the Milky Way halo may well be poorly approximated by a standard Maxwellian halo, the analysis technique employed should not pre-suppose an exponentially declining form for $\mathrm{d} R / \mathrm{d} E_{\mathrm{R}}$. In fact it would be useful, if possible, to formulate an analysis technique which decouples the WIMP cross-section from the assumed WIMP speed distribution.

\section{ACKNOWLEDGEMENTS}

A.M.G. was supported by PPARC and acknowledges use of the Starlink computer system at Queen Mary, University of London.

Whilst completing this work a preprint appeared by the CRESST collaboration [17, in which they mention algorithms which they have developed to extract exclusion limits from their experiment.

[1] K. M. Ashman, Publ. Astron. Soc. Pac., 104, 1109 (1992); C. J. Kochanek, Astophys. J. 445, 559 (1995).

[2] J. R. Primack, B. Sadoulet and D. Seckel, Ann. Rev. Nucl. Part. Sci., B38, 751 (1988).

[3] G. Jungman, M. Kamionkowski and K. Griest, Phys. Rep. 267, 195 (1996).

[4] R. Bernabei et. al. Phys. Lett. B389, 757 (1996); ibid B408, 439 (1997); ibid B424, 195 (1998); ibid B450, 448 (1999); ibid B480, 23 (2000).

[5] G. Gerbier, J. Mallet, L. Mosca and C. Tao, astroph/9710181; astro-ph/9902194.

[6] R. Abusaidi et. al., Phys. Rev. Lett. 84, 5699 (2000).

[7] S. Golwala, PhD thesis, University of California Berkeley, (2000).

[8] R. Schnee, proceedings of 'COSMO 2000', Cheju Island, Korea, Sept. 4-8 (2000).

[9] L. Baudis et. al., Phys. Rev D 59, 022001 (1999).

[10] A. Morales et. al., Phys. Lett. B 489, 268 (2000); S. Cebrian et. al. Nucl. Phys. B Proc. Suppl. 95, 229 (2001).

[11] G. J. Feldman and R. D. Cousins, Phys. Rev. D 57, 3873 (1998).

[12] see e.g. Gradshteyn and Ryzkik, 'Tables of Integral, Series and Products'.

[13] J. D. Lewin and P. F. Smith, Astropart. Phys. 6, 87 (1996).

[14] J. R. Ipser and P. Sikivie, Phys. Lett. B 291, 288 (1992); P. Sikivie, I. I. Tkachev and Y. Wang, Phys. Rev. Lett. 75, 2911 (1995); Phys. Rev. D 56, 1863 (1997).
[15] A. M. Green, Phys. Rev. D 63103003 (2001); G. Gelmini and P. Gondolo, Phys. Rev. D 64, 023504 (2001).

[16] B. Moore, to appear in the proceedings of IDM2000 'Third international workshop on the identification of dark matter' ed. N Spooner, astro-ph/0103094; B. Moore et. al. to appear in Phys. Rev. D, astro-ph/0106271.

[17] M. Altmann et. al. paper contributed to 'The X International Symposium on Lepton and Photon Interactions at High Energies', July 2001, Rome, astro-ph/0106314. 